Article

\title{
Effects on Nitric Oxide Production of Urolithins, Gut-Derived Ellagitannin Metabolites, in Human Aortic Endothelial Cells
}

\author{
Valentina Spigoni ${ }^{1, \dagger}$, Pedro Mena ${ }^{2, \dagger}$, Monia Cito ${ }^{1}$, Federica Fantuzzi ${ }^{1,3}$, Riccardo C. Bonadonna ${ }^{1,3}$, \\ Furio Brighenti ${ }^{2}$, Alessandra Dei Cas ${ }^{1,3}$ and Daniele Del Rio ${ }^{2, *}$ \\ 1 Endocrinology and Metabolism, Department of Clinical and Experimental Medicine, University of Parma, \\ Parma 43126, Italy; valentina.spigoni@unipr.it (V.S.); monia.cito@hotmail.it (M.C.); \\ federica.fantuzzi1@gmail.com (F.F.); riccardo.bonadonna@unipr.it (R.C.B.); \\ alessandra.deicas@unipr.it (A.D.C.) \\ 2 The Laboratory of Phytochemicals in Physiology, Department of Food Science, University of Parma, \\ Parma 43125, Italy; pedromiguel.menaparreno@unipr.it (P.M.); furio.brighenti@unipr.it (F.B.) \\ 3 Division of Endocrinology, Azienda Ospedaliero-Universitaria of Parma, Parma 43126, Italy \\ * Correspondence: daniele.delrio@unipr.it; Tel.: +39-0521-903-830; Fax: +39-0521-903-832 \\ + These authors contributed equally to this work.
}

Academic Editor: Rafael Llorach

Received: 13 June 2016; Accepted: 29 July 2016; Published: 2 August 2016

\begin{abstract}
The consumption of foodstuffs yielding circulating compounds able to maintain endothelial function by improving nitric oxide (NO) bioavailability can be considered as an effective strategy for cardiovascular disease prevention. This work assessed the in vitro effects of urolithin A, urolithin $\mathrm{B}$, and urolithin B-glucuronide, ellagitannin-derived metabolites of colonic origin, on NO release and endothelial NO synthase (eNOS) activation in primary human aortic endothelial cells (HAECs). Urolithins were tested both individually at $15 \mu \mathrm{M}$ and as a mixture of $5 \mu \mathrm{M}$ each, at different time points. The biotransformation of these molecules in cell media due to cell metabolism was also evaluated by UHPLC-MS ${ }^{\mathrm{n}}$. The mix of urolithins at $5 \mu \mathrm{M}$ significantly increased nitrite/nitrate levels following $24 \mathrm{~h}$ of incubation, while single urolithins at $15 \mu \mathrm{M}$ did not modify NO bioavailability. Both the mix of urolithins at $5 \mu \mathrm{M}$ and urolithin B-glucuronide at $15 \mu \mathrm{M}$ activated eNOS expression. All urolithins underwent metabolic reactions, but these were limited to conjugation with sulfate moieties. This study represents a step forward in the understanding of cardiovascular health benefits of ellagitannin-rich foodstuffs and backs the idea that peripheral cells may contribute to urolithin metabolism.
\end{abstract}

Keywords: urolithin; ellagic acid; microbiota metabolites; endothelial cells; endothelial nitric oxide synthase; endothelial function; atherosclerosis; peripheral metabolism

\section{Introduction}

The endothelium is a key regulator of vascular homeostasis, representing not only a selective barrier, but also modulating circulating blood cell adhesion, smooth muscle cell proliferation, and inflammation. In addition, it plays a crucial role in the maintenance of vascular tone by regulating the fine-tuned balance between vasodilation and vasoconstriction [1]. Nitric oxide (NO) is the most important endothelium-dependent vasodilator produced by the endothelial nitric oxide synthase (eNOS) in blood vessels. Decreased eNOS activation and the subsequent reduction in NO bioavailability are the main determinants of endothelial dysfunction, an early step in the development 
of atherosclerosis [2]. Endothelial dysfunction is a reversible process and effective strategies in maintaining or improving vascular homeostasis are of critical interest for cardiovascular disease (CVD) prevention.

Phenolic compounds may exert protective actions against atherosclerosis and other CVDs [3-6]. Recently, a randomized, controlled, double-masked trial showed a significant reduced risk of coronary heart disease (CHD), myocardial infarction, and death from CHD and CVD after bi-daily ingestion of cocoa flavanols [7]. This same study reported a significant improvement in endothelial function (measured as flow-mediated vasodilation (FMD)). Consumption of polyphenol-rich cranberry juice also improved endothelial function in patients with coronary artery disease [8] and healthy men [9]. While it may seem obvious to ascribe the effects of foodstuffs rich in (poly)phenolic compounds on endothelial function to their phenolic fraction, the relationship between the metabolites in circulation and the observed effects is not usually tackled [8]. Interestingly, current literature accounts for a positive change in addressing this issue [9]. On the other hand, cell-based evidence on the modulation of endothelial function by phenolic compounds is far from explaining the effects observed in humans. Different cell studies carried out with phenolic compounds have shown induced NO release through a promotion of eNOS expression in human endothelial cells $[10,11]$. However, metabolic transformations or the dose of circulating phenolic metabolites have not been really taken into account in the past. In most of the cases, in vitro studies have applied molecules never appearing in vivo, or concentrations far from those achievable in the context of a normal diet. Currently, the number of cell studies performed with phenolic circulating metabolites at physiological concentrations is growing and outcomes point to a modulated vascular reactivity and improved NO bioavailability [12,13].

Ellagitannins are hydrolyzable tannins present in pomegranate, walnuts, berries such as raspberries, strawberries, and blackberries, and oak-aged wines [14]. Once consumed, ellagitannins are hydrolyzed into ellagic acid, which is further metabolized by the intestinal microbiota to produce urolithins [15]. Urolithins are hydroxylated $6 H$-dibenzo[ $b, d]$ pyran-6-one structures that may undergo colonic/hepatic transformation upon absorption and appear in circulation as sulfate, glucuronide, and methyl metabolites at concentrations in the low micromolar-high nanomolar range [14-16]. Although the evidence is limited, some experimental studies have demonstrated the anti-inflammatory and anti-proliferative effects of urolithins, as well as the ability of some food matrixes rich in ellagitannins to improve endothelial function [17-27]. Nevertheless, the potential role of urolithins in modulating NO-related endothelial cell function has been scarcely investigated to date [28].

The aim of this work was to assess the in vitro effects of urolithin A (Uro A, dihydroxy-urolithin), urolithin B (Uro B, monohydroxy-urolithin), and urolithin B-glucuronide (Uro Bgluc) on endothelial function, in terms of eNOS activation and NO release, in primary human aortic endothelial cells (HAECs). These molecules were tested both individually, at $15 \mu \mathrm{M}$, and as a mixture of $5 \mu \mathrm{M}$ each. The biotransformations of these ellagitannin-derived metabolites in the tested cell model, as a consequence of cell metabolism, were also evaluated.

\section{Results}

\subsection{NO Production in Human Aortic Endothelial Cells}

To establish whether urolithins could modulate vascular reactivity in a non-inflammatory scenario, the production of $\mathrm{NO}$ was studied in non-stimulated conditions. Urolithins were added individually at $15 \mu \mathrm{M}$, in line with previous works [18], and pooled at $5 \mu \mathrm{M}$ each $(5 \mu \mathrm{M}$ of Uro A, $5 \mu \mathrm{M}$ of Uro B, and $5 \mu \mathrm{M}$ of Uro Bgluc, named "mix"), similarly to Mele et al. [25], to obtain the same final metabolite concentration.

Endothelial NOS activation by "classical" cues (such as insulin and bradykinin) is known to occur acutely (after a few minutes) in endothelial cells $[29,30]$. In addition, since NO is a gaseous molecule with a short half-life, nitrite $\left(\mathrm{NO}^{2-}\right)$ and nitrate $\left(\mathrm{NO}^{3-}\right)$ levels-which represent the stable degradation 
products of $\mathrm{NO}$ accumulating in supernatants of cell culture-were assessed at $5 \mathrm{~min}$ and at $24 \mathrm{~h}$ as an integrated measure of NO production [31-33].

None of the single urolithins at $15 \mu \mathrm{M}$ modified NO metabolite production in HAECs following both $5 \mathrm{~min}$ and $24 \mathrm{~h}$ of incubation, compared to vehicle culture conditions (Figure 1). However, the mix of urolithins significantly increased nitrite + nitrate levels in HAECs following $24 \mathrm{~h}$ of incubation $(p<0.01)$. This may be the result of the higher efficacy of urolithins pooled at $5 \mu \mathrm{M}$, than when tested individually at $15 \mu \mathrm{M}$. On the other hand, it is worth mentioning the high variability observed among independent experiments, which could be attributed to the nature of these primary cells.
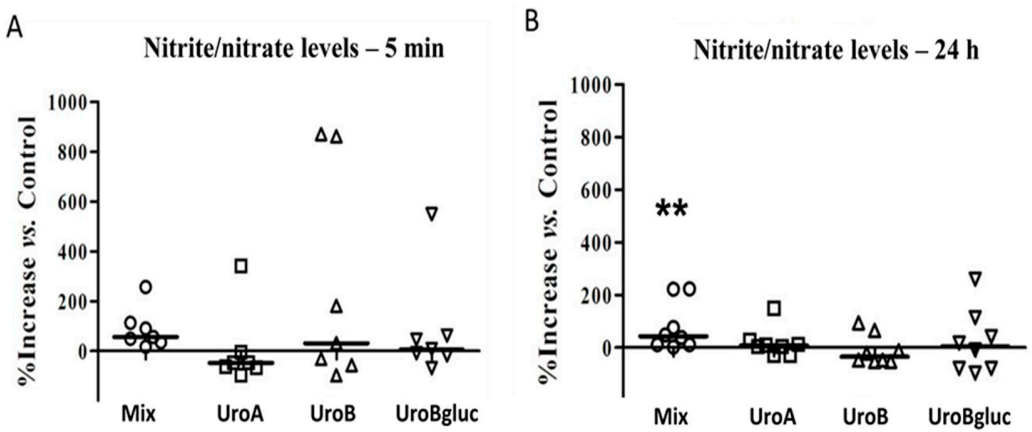

Figure 1. Variation in nitrite/nitrate levels in the supernatant of HAECs cultured at $5 \mathrm{~min}(\mathrm{~A})$ and $24 \mathrm{~h}$ (B) with respect to control condition $(0.1 \% \mathrm{DMSO})$. Mix: urolithin mix at $5 \mu \mathrm{M}$ each; uro A: urolithin A; uro B: urolithin B; Uro Bgluc: urolithin B-glucuronide. $n=7$; ${ }^{* *}$ significant at $p<0.01$ vs. control. Each experimental condition is represented by a specific symbol (Mix: circles, Uro A: squares, Uro B: triangles, Uro Bgluc: inverted triangles) and bars represent the median values.

\section{2. eNOS Activation in Human Aortic Endothelial Cells}

In order to clarify the role of eNOS in the observed NO secretion, eNOS activation at the same time points ( $5 \mathrm{~min}$ and $24 \mathrm{~h}$ ) was assessed. Exposure to mixed urolithins increased eNOS activation at $5 \mathrm{~min}$ (1.8-fold increase vs. control) but not at $24 \mathrm{~h}$ (Figure 2), justifying the increase in NO levels at the later time point $(24 \mathrm{~h})$. Uro A and Uro B at $15 \mu \mathrm{M}$ did not exert any statistically significant effect on eNOS expression either at $5 \mathrm{~min}$ or at $24 \mathrm{~h}$. In contrast, a significant 2.4-fold increase in eNOS activation compared to the control was observed when HAECs were incubated in the presence of Uro Bgluc $15 \mu \mathrm{M}$ for $5 \mathrm{~min}$.


Figure 2. eNOS activation in HAECs cultured at $5 \mathrm{~min}$ (A) and $24 \mathrm{~h}$ (B) with respect to control condition (0.1\% DMSO). eNOS activation was assessed by Western blotting and expressed as the ratio of phosphorylated-eNOS (p-eNOS) to total eNOS protein expression. Beta-actin has been used as loading control. One representative experiment is reported, bars represent median values $(n=7)$. Mix, urolithin mix at $5 \mu \mathrm{M}$ each; uro A, urolithin A; uro B, urolithin B; and Uro Bgluc, urolithin B-glucuronide. ${ }^{* *}$ significant at $p<0.01$ vs. control. 


\subsection{In Vitro Metabolism of Urolithins in HAECs}

Cellular metabolism is a key point to be considered when dealing with the biological activity of phenolic compounds, since the ability of some cell types to uptake and metabolize phenolic compounds or their metabolites may influence the biological effects of the tested parent compounds. Gastrointestinal tract cells are recognized to be pivotal in the absorption, metabolism, and bioactivity of phenolics [4,6], but other cell types may also exert metabolic structural modifications. This peripheral metabolism of phenolic compounds, and in particular of their circulating metabolites, has been scarcely studied [34]. Recently, some progress has been achieved in the case of urolithins by using cardiomyocytes and cardiac fibroblasts [26], and macrophages and human umbilical vein endothelial cells (HUVECs) [25]. However, the intracellular metabolism of urolithins by human aortic endothelial cells was still not reported.

In order to verify the peripheral metabolism of urolithins in HAECs and to get insights into the real molecules behind the effects observed on NO bioavailability, cell media of the performed experiments were analyzed at the beginning and end of each incubation time. Culture media and non-incubated cell media were used to test urolithin stability and as the control, respectively. Different transformations that might take place (conjugation with sulfate, glucuronide, methyl, glutathione, and cysteine moieties, quinone formation, and (de)hydroxylation) were monitored by a specific and sensitive ultra-high performance liquid chromatography coupled to mass spectrometry (UHPLC-MS ${ }^{n}$ ) analysis [25]. Four newly formed metabolites were identified according to their fragmentation patterns and retention times (Table 1). All urolithins underwent metabolic reactions, but were basically limited to conjugation with sulfate moieties (Figure 3). Unfortunately, newly formed metabolites were not quantified as reference compounds were not commercially available. Thus, peak area values were considered when comparisons were made.

Both Uro A and Uro B were stable under cell culture conditions, while Uro Bgluc underwent a weak deglucuronidation $(\sim 1 \%)$. Despite all the tested molecules undergoing metabolic reactions, the concentration of the incubated molecules, both individually and mixed, remained almost unaltered after $24 \mathrm{~h}(p>0.05)$, indicating a quite negligible conversion rate. The formation of sulfated metabolites in the cell culture was detected at $24 \mathrm{~h}$ but not at $5 \mathrm{~min}$ after urolithin incubation. In the case of Uro B-sulfate, a higher rate of sulfation was observed for Uro B than for Uro Bgluc (about nine-fold higher, $p<0.001$ ) (Figure 4). With regard to metabolites derived from Uro A, mono-sulfated isomers were almost equally concentrated, while Uro A-disulfate was detected only at trace levels. Finally, it should be noted that the co-presence in the mix of the three urolithins (Uro B, Uro Bgluc, and Uro A) did not alter the metabolic activity of HAECs, since the metabolites identified when the cells were incubated with single compounds were also detected after exposure to the mix.

Table 1. Urolithins detected in endothelial cells.

\begin{tabular}{lccccc}
\hline Compound & Derived Metabolite & $\begin{array}{c}\text { Retention } \\
\text { Time (min) }\end{array}$ & $\begin{array}{c}{[\mathbf{M}-\mathbf{H}]^{-}} \\
(\mathbf{m} / \mathbf{z})\end{array}$ & $\begin{array}{c}\mathbf{M S}^{\mathbf{2}} \text { Ion } \\
\text { Fragments }(\mathbf{m} / \mathbf{z})\end{array}$ & $\begin{array}{c}\mathbf{M S}^{\mathbf{3}} \text { Ion } \\
\text { Fragments }(\mathbf{m} / \mathbf{z})\end{array}$ \\
\hline \multirow{3}{*}{ Urolithin B } & Uro B & 4.50 & 211 & 167,182 & \\
& Uro B-sulfate & 3.71 & 291 & $211^{1}$ & 167 \\
& Uro B-glucuronide & 2.45 & 367 & 211,175 & 167 \\
\multirow{3}{*}{ Urolithin A } & 3.19 & 227 & $183,159,199$ & 183 \\
& Uro A & 1.58 & 307 & 227 & 183 \\
& Uro A-sulfate isomer 1 & 1.86 & 307 & 227 & 227 \\
\hline
\end{tabular}

${ }^{1} \mathrm{MS}^{2}$ Ions in italic were those subjected to $\mathrm{MS}^{3}$ fragmentation. 


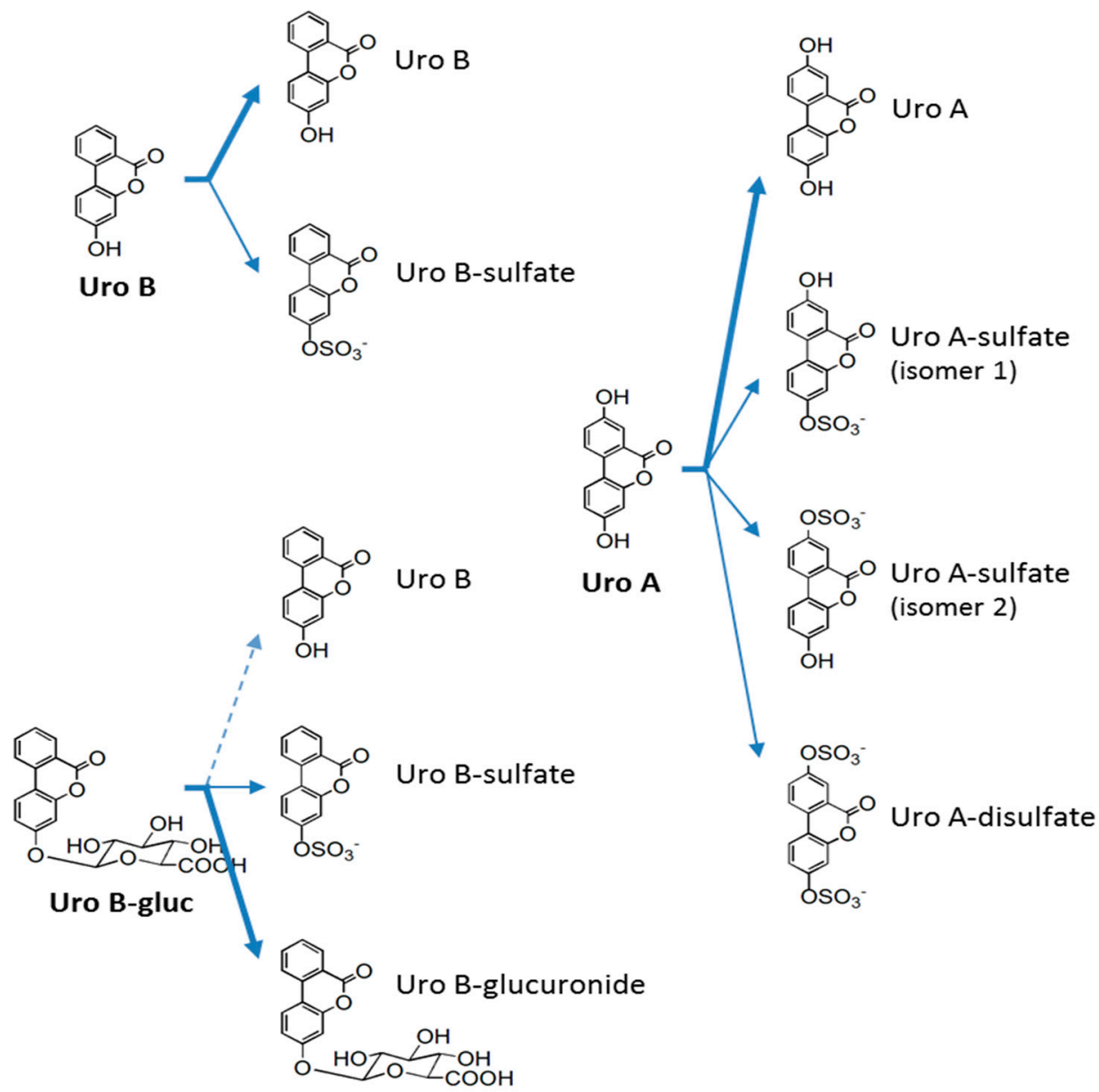

Figure 3. Metabolic biotransformations occurring in human aortic endothelial cells and compounds present contemporaneously in the cell media at $24 \mathrm{~h}$. Bold arrows indicate major pathways, which correspond to incubated urolithins; normal arrows, newly-formed metabolites; and dotted arrows, spontaneous deglucuronidation due to cell media conditions.
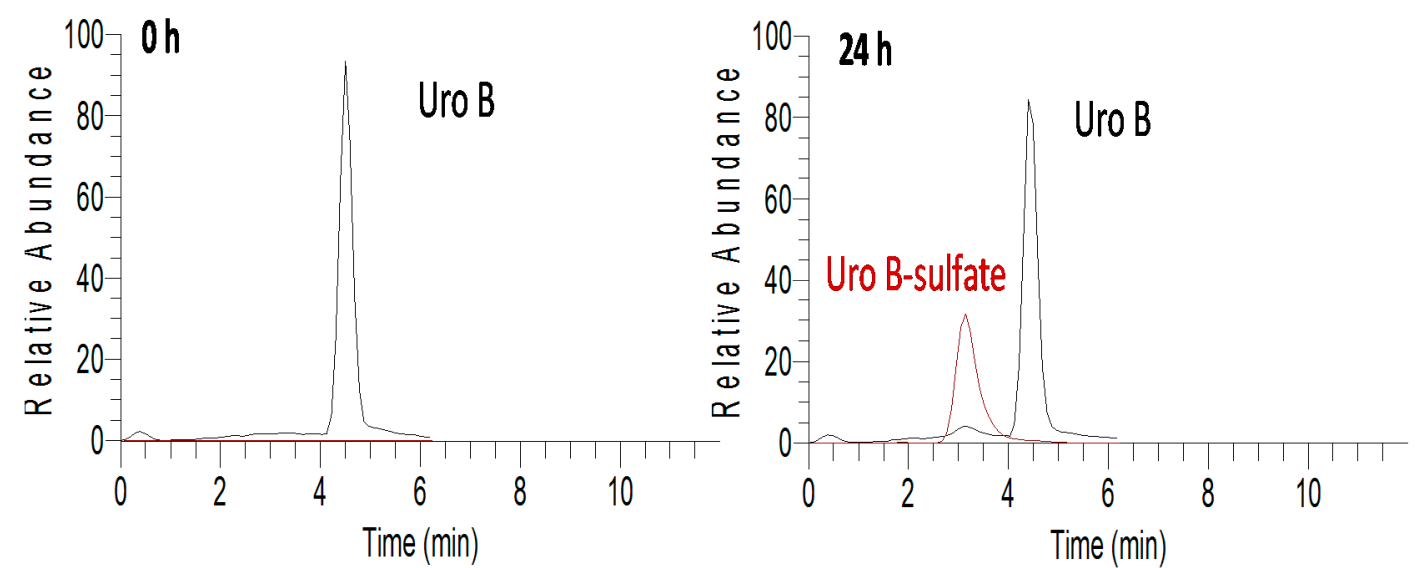

Figure 4. Chromatograms of compounds present in cell media at 0 and after $24 \mathrm{~h}$ of incubation with Uro B, extracted in selected ion-monitoring mode. Areas of the metabolites should not be considered as quantitative indicators since ionization efficiencies of the individual compounds may be different. 


\section{Discussion}

Impairment in eNOS activation preludes endothelial dysfunction and atherosclerosis. Therefore, the demonstration of a benefit of ellagitannin-derived metabolites on endothelial function could be considered a mechanism through which dietary ellagitannins may contribute to protection against atherosclerotic processes. An in vivo study revealed that pomegranate fruit extract rich in ellagitannins significantly decreased the expression of vascular inflammation markers (thrombospondin and cytokines) and increased plasma NO levels in obese Zucker rats [17]; while in another study, the administration of $100-300 \mathrm{mg} / \mathrm{kg} /$ day for four weeks of pomegranate juice extract to diabetic rats treated with angiotensin II decreased arterial blood pressure and improved biomarkers linked to oxidative stress [35]. While some in vitro studies have shown anti-atherosclerotic effects of ellagitannins in endothelial cells by exposing culture media to full plant extracts or to single parent plant compounds at rather high concentrations [15], the present study was the first in assessing the effects of a mix of circulating ellagitannin metabolites at physiologically attainable concentrations on endothelial cell function. Results showed how a mixture of $5 \mu \mathrm{M}$ of urolithins A, B, and B-glucuronide, the most representative ellagitannin-derived metabolites, increased NOx bioavailability by eNOS activation in human aortic endothelial cells. The fact that the mixture of urolithins seemed to be more bioactive in improving NO release compared to its individual constituent compounds is of critical interest. It may suggest that the reported benefits of an ellagitannin-rich diet could be due to the activities of metabolites working in combination, as it would potentially occur following dietary exposure, and not merely as the exact consequence of a single metabolite. This assumption has been recently proposed in an in vitro study in which combinations of flavonoid-derived metabolites significantly reduced TNF- $\alpha$ to a greater extent than their precursors or single metabolites alone [36]. Although the effects of polyphenol metabolites on NO release might be magnified in insulin-stimulated conditions [37], we decided to carry out the experiments in a basal unstimulated experimental setting, in order to avoid potential confounding factors. We cannot exclude that the presence of insulin might have amplified the observed results. Furthermore, it must be considered that nitrite/nitrate assessment, although commonly used, is an indirect measure of NO levels, and therefore per se constitutes a limitation.

Uro Bgluc at $15 \mu \mathrm{M}$ increased eNOS protein activation in the tested cell model. However, increased eNOS activation following Uro Bgluc incubation was not accompanied by an increase in NO levels. This effect is not easily justified, and one can speculate that the urolithin mix could lead to more persistent enzyme activation than Uro Bgluc, with a subsequent major increase in NO production. Hypothetically, this persistent activation of eNOS would have been detectable in the time lapse between the $5 \mathrm{~min}$ and the $24 \mathrm{~h}$ time points, which was not investigated in this study. The $15 \mu \mathrm{M}$ concentration of metabolites used in this study is comparable to that obtained in a study after the ingestion of $1 \mathrm{~L}$ of pomegranate juice containing $4.37 \mathrm{~g}$ of punicalagins for five days, in which plasma urolithin levels reached a maximum concentration of $18.6 \mu \mathrm{M}$ [38]. Although lower concentrations of circulating Uro Bgluc are expected after normal consumption of ellagitannin-rich foodstuffs, these results are of clinical interest since they may generate interesting prospects in terms of cardiovascular health promotion and future research. Nevertheless, it should be kept in mind that current evidence accounts for a large interand intra-individual variability in the levels of circulating urolithins, indicative of differences in the colonic microbiota responsible for ellagitannin degradation [39]. This fact may certainly hamper the settings of mean values for ellagitannin-derived circulating metabolites, as well as dietary guidelines for ellagitannin consumption.

Importantly, our study was performed in human cultured endothelial cells (HAECs), which represent a primary cell population directly exposed to circulating urolithin metabolites and involved in the pathophysiology of atherosclerosis. In the same cellular model and in accordance with our data, a very recent work showed that Uro A attenuated ox-LDL-induced endothelial dysfunction by an up-regulation of NO expression and eNOS mRNA expression [28]. In addition, Uro A and its 
glucuronide Uro A-glucuronide showed anti-inflammatory effects by reducing monocyte adhesion, endothelial cell migration and expression of pro-inflammatory chemokines [18].

This mounting evidence may serve to establish the role of ellagitannin metabolites in the promotion of endothelial health. By showing positive effects of these metabolites in improving vascular endothelial cell function in vitro, these results also expand previous knowledge of the anti-inflammatory and chemopreventive effects of urolithins [18-22,24-27]. Actually, most of the studies conducted so far clearly demonstrated the anti-inflammatory effects of urolithins in THP-1 [25] and RAW macrophages [40]. Importantly, in an in vivo study Uro A supplementation was able to abrogate the NO production by avoiding iNOS induction in the colonic mucosa of a colitis rat model [24]. The putative molecular pathway underlying the capacity of urolithins to increase $\mathrm{NO}_{\mathrm{x}}$ production is beyond the scope of our study. However, on a purely speculative base, urolithins contain hydroxyl groups, which may potentially generate ROS through auto-oxidation. This, in turn, may induce the activation of Src kinases (i.e., Akt, AMPK, and eNOS) leading to NOx production, as previously demonstrated for other polyphenols, i.e., hesperidin [41], epigallocatechin gallate [31], and red wine polyphenols [42], as a putative mechanism for improved endothelial function in vitro.

In order to fully elucidate the real metabolites behind the effects showed in the HAECs, the possible biotransformations of urolithins in cell media were assessed. Actually, urolithins have been shown to undergo extensive metabolism in peripheral cells and not only at colonic and hepatic levels $[25,26]$. Our research group has described how both Uro A and Uro B are sulfated by both HUVECs and THP-1-derived macrophages [25]. The modifications carried out by cardiomyocytes and cardiac fibroblasts include, besides sulfation, glucuronidation and, in the case of Uro Bgluc, deglucuronidation [26]. The present results indicate sulfation as the prevalent modification taking place in urolithins incubated with HAECs. Regarding all these outcomes, cell-mediated sulfation seems to be a common metabolic step undergone by urolithins in peripheral cells of the cardiovascular system. Nonetheless, with glucuronidation considered to be the main reaction occurring in vivo after ellagitannin consumption [38,43], deglucuronidation must happen before sulfation, at least of Uro Bgluc. Obviously, the quantitative levels or proportions of these newly formed metabolites, even in relation to their precursors, should be taken with caution, as without analytical standards one cannot guarantee ionization efficiencies of the structures will be similar.

Metabolic transformations in peripheral cells may be relevant for the understanding of the compounds and mechanisms responsible for the bioactivity of phenolic compounds. In the particular case of urolithins, different phase II conjugates (with glucuronide, sulfate, and methyl moieties) of the same chemical scaffold may cause different biological responses [44,45]. Deglucuronidation of quercetin-glucuronide has been reported to be a crucial, positive step in the anti-inflammatory properties of quercetin on lipopolysaccharide-stimulated macrophages [46,47]. On the contrary, quercetin deglucuronidation reduces eNOS protein and gene expression in HUVECs and, thus, might cause a potentially deleterious impairment in the endothelial function response [48]. However, these metabolic reactions should not be considered as generalized for all phenolic metabolites. For instance, no deglucuronidation was observed when differently activated macrophages were exposed to naringenin-glucuronide isomers (naringenin-7-O-glucuronide and narigenin-4'-O-glucuronide) at concentrations coherent with dietary exposure [49]. Similarly, major flavan-3-ol metabolites ((-)-epicatechin-3'-glucuronide, (-)-epicatechin-3'-sulfate, 3'-O-methyl-(-)-epicatechin-5-sulfate, and $3^{\prime}-O$-methyl-(-)-epicatechin-7-sulfate) were not metabolized when incubated with HUVECs, while exposure of free epicatechin to HUVECs entailed its intracellular metabolism to 3'-O-methyl-(-)-epicatechin-7-sulfate and 3'-O-methyl-(-)-epicatechin-7-glucuronide [34]. 


\section{Materials and Methods}

\subsection{Chemicals}

Uro A, Uro B, and Uro Bgluc were provided by Olivier Dangles (INRA, Avignon, France), and synthesised according to what described in Tognolini et al. [50].

\subsection{Cell Culture}

HAECs (purchased from GIBCO Life Technologies, Carlsbad, CA, USA) were cultured in complete EGM-2 Bullet Kit (Lonza, Basel, Switzerland) at $37{ }^{\circ} \mathrm{C}$ in a $5 \% \mathrm{CO}_{2}$ humidified incubator and used at passages 3-7 in all the experiments. HAEC were seeded in T25 flasks at a density of $1 \times 10^{4} \mathrm{cells} / \mathrm{cm}^{2}$. On reaching $70 \%-80 \%$ confluence, cells were cultured overnight in serum free-media and then incubated with urolithins or with vehicle (0.1\% DMSO as negative control) for $5 \mathrm{~min}$ and $24 \mathrm{~h}$. Uro A, Uro B and Uro Bgluc were incubated at a concentration of $15 \mu \mathrm{M}$. A mixture of urolithins at $5 \mu \mathrm{M}$ each was also added to the culture medium in order to test the combined effects of metabolites.

\subsection{Nitrite Analysis}

Total nitrite (nitrate and nitrite) produced by cells was measured in the supernatants by using a nitrate/nitrite assay kit (Cayman Chemical Company, Ann Arbor, MI, USA), following manufacturer's instructions. This assay determines NO concentrations based on the enzymatic conversion of nitrate to nitrite by nitrate reductase. The reaction is followed by colorimetric detection of nitrite. A standard curve was performed to quantitate nitrite concentration in each assay. Seven independent experiments were performed in triplicate for each condition and results were expressed as nitrite percentage increase in urolithin-treated cells compared to vehicle.

\subsection{Total Protein Extraction}

Following treatments, HAEC were washed twice with PBS, detached from the flasks by using trypsin-EDTA, centrifuged and stored at $-20{ }^{\circ} \mathrm{C}$. Mammalian Protein Extraction Reagent (M-PER, Thermo Fisher Scientific, Milano, Italy) containing Halt ${ }^{\mathrm{TM}}$ Protease and Phosphatase Inhibitor (Single-Use Cocktail, Thermo Scientific) was added to the cell pellets and proteins were extracted by gentle shacking for $10 \mathrm{~min}$ followed by centrifugation at $13,000 \times g$ for $15 \mathrm{~min}$ in order to remove cell debris. Protein quantification was determined according to the Bio-Rad Protein Assay (Bio-Rad Laboratories, Hercules, CA, USA) using bovine serum albumin (BSA) as standard.

\subsection{Western Blotting}

A total of $40 \mu \mathrm{g}$ of proteins were separated by $4 \%-12 \%$ pre-cast polyacrylamide gel (NuPAGE ${ }^{\circledR}$ Novex ${ }^{\circledR} 4 \%-12 \%$ Bis-Tris Gels, Thermo Scientific) and transferred to nitrocellulose filters. Membranes were blocked with 5\% skim milk (Membrane Blocking Agent, GE Healthcare, Chalfont St Giles, UK) in Tris-buffered saline solution containing $0.1 \%$ Tween-20 and incubated overnight at $4{ }^{\circ} \mathrm{C}$ with rabbit anti-human phospho-eNOS ${ }^{S e r 1177}$ (1:1000), eNOS (1:1000) or $\beta$-actin $(1: 10,000)($ Cell Signaling Technology, Boston, MA, USA) antibodies. Membranes were then incubated with Horseradish peroxidase-labeled goat anti-rabbit secondary antibody (Cell Signaling) and detected by chemiluminescence using ECL western Blotting Detection Reagent (GE Healthcare, Chalfont St Giles, UK). Images were scanned and quantified using ImageJ software (Version 1.48, National Institutes of Health, MD, USA). Activation of eNOS was expressed as a ratio of p-eNOS to eNOS (which detects both phosphorylated and non-phosphorylated forms).

\subsection{UHPLC-MS ${ }^{n}$ Analysis of Cell Media}

Cell media supernatants were collected and analyzed by UHPLC-MS ${ }^{n}$ to determine the stability and intracellular metabolism of the urolithins in cell culture. Cell media was extracted according 
to Sala et al. [26] and analyzed according to Mele et al. [25]. Briefly, samples were analysed using an Accela UHPLC 1250 equipped with a linear ion trap-mass spectrometer (LTQ XL, Thermo Fisher Scientific Inc., San Jose, CA, USA) fitted with a heated-electrospray ionization probe (H-ESI-II; Thermo Fisher Scientific Inc.). Separations were performed using a XSELECTED HSS T3 $(50 \times 2.1 \mathrm{~mm}), 2.5 \mu \mathrm{M}$ particle size (Waters, Milford, MA, USA), with an injection volume of $5 \mu \mathrm{L}$, column oven temperature of $30{ }^{\circ} \mathrm{C}$ and elution flow rate of $0.2 \mathrm{~mL} / \mathrm{min}$. The initial gradient was $75 \%$ of $0.1 \%$ aqueous formic acid and $25 \%$ acetonitrile (in $0.1 \%$ formic acid), reaching $80 \%$ acetonitrile at $6 \mathrm{~min}$. The MS conditions included: capillary temperature of $275^{\circ} \mathrm{C}$ and source heater temperature of $250^{\circ} \mathrm{C}$, sheath gas flow of 40 units, auxiliary and sweep gas of five units, source voltage of $3 \mathrm{kV}$ and capillary voltage and tube lens of -5 and $-68 \mathrm{~V}$, respectively. Analyses were carried out using full scan, data-dependent MS $^{3}$ scanning from $m / z 100$ to 600, with collision induced dissociation (CID) equal to 35 (arbitrary units). Pure helium gas was used for CID. Data processing was performed using Xcalibur software (Version 2.2) from Thermo Scientific.

\subsection{Statistical Analysis}

Due to significant deviations from normality, the exact version of Friedman test was used to investigate differences among culture conditions. Differences between pairs of groups were identified using paired Wilcoxon test with Bonferroni correction for multiplicity $(p<0.0125)$.

Statistical significance was declared at $p<0.05$ (two-sided). SPSS 21 (IBM, Armonk, NY, USA) and Prism 4 (Graphpad, La Jolla, CA, USA) were used for statistical analyses and graphs.

\section{Conclusions}

This study represents a step forward in the understanding of the cardiovascular health benefits of ellagitannin-rich foodstuffs. It evidenced how urolithin B glucuronide at $15 \mu \mathrm{M}$ and a mixture of urolithins A, B, and B-glucuronide at $5 \mu \mathrm{M}$ each can be effective in improving aortic endothelial cell function in vitro. These results also support the idea that peripheral cells may contribute significantly to urolithin metabolism.

Although promising, these in vitro results on the biological activity of urolithins should be cautiously translated to in vivo physiological conditions. Future randomized, controlled human interventions, properly designed to take into account the late production of these ellagitannin-derived metabolites, are required to fully define the cardioprotective features of ellagitannin-rich foodstuffs. Similarly, novel dynamic models of cell culture adhering to transient concentrations of phenolic metabolites should be investigated.

Acknowledgments: This study was supported by a research grant from the US National Processed Raspberry Council (Contract No. 2015-7) and Grants from the University of Parma to A.D.C. and R.C.B.; P.M. was funded by a grant of the Postdoctoral Fellowship Program from the Fundación Séneca (Murcia Region, Spain).

Author Contributions: D.D.R. and A.D.C. conceived and designed the experiments; V.S., P.M., M.C., and F.F. performed the experiments; V.S., A.D.C., and P.M. analyzed the data; D.D.R., F.B., R.C.B. and A.D.C. contributed reagents/materials/analysis tools; V.S., P.M., and D.D.R. wrote the paper.

Conflicts of Interest: The authors declare no conflict of interest. The funding sponsors had no role in the design of the study; in the collection, analyses or interpretation of data; in the writing of the manuscript, and in the decision to publish the results.

\section{Abbreviations}

The following abbreviations are used in this manuscript:

$\begin{array}{ll}\text { CHD } & \text { coronary heart disease } \\ \text { CVD } & \text { cardiovascular disease } \\ \text { eNOS } & \text { endothelial nitric oxide synthase } \\ \text { FMD } & \text { flow-mediated vasodilation } \\ \text { HAEC } & \text { human aortic endothelial cells } \\ \text { HUVEC } & \text { human umbilical vein endothelial cells } \\ \text { NO } & \text { nitric oxide }\end{array}$


UHPLC-MS $\quad$ ultra-high performance liquid chromatography coupled to mass spectrometry

Uro A

urolithin A

Uro B

Uro Bgluc

urolithin B

References

1. Sessa, W.C. Molecular control of blood flow and angiogenesis: Role of nitric oxide. J. Thromb. Haemost. 2009, 7 (Suppl. S1), 35-37. [CrossRef] [PubMed]

2. Forstermann, U.; Munzel, T. Endothelial nitric oxide synthase in vascular disease: From marvel to menace. Circulation 2006, 113, 1708-1714. [CrossRef] [PubMed]

3. Auger, C.; Said, A.; Nguyen, P.N.; Chabert, P.; Idris-Khodja, N.; Schini-Kerth, V.B. Potential of food and natural products to promote endothelial and vascular health. J. Cardiovasc. Pharmacol. 2016, 68, 11-18. [CrossRef] [PubMed]

4. Del Rio, D.; Rodriguez-Mateos, A.; Spencer, J.P.E.; Tognolini, M.; Borges, G.; Crozier, A. Dietary (poly)phenolics in human health: Structures, bioavailability, and evidence of protective effects against chronic diseases. Antioxid. Redox Signal. 2013, 18, 1818-1892. [CrossRef] [PubMed]

5. Zanotti, I.; Dall'Asta, M.; Mena, P.; Mele, L.; Bruni, R.; Ray, S.; Del Rio, D. Atheroprotective effects of (poly)phenols: A focus on cell cholesterol metabolism. Food Funct. 2015, 6, 13-31. [CrossRef] [PubMed]

6. Rodriguez-Mateos, A.; Vauzour, D.; Krueger, C.G.; Shanmuganayagam, D.; Reed, J.; Calani, L.; Mena, P.; Del Rio, D.; Crozier, A. Bioavailability, bioactivity and impact on health of dietary flavonoids and related compounds: An update. Arch. Toxicol. 2014, 88, 1803-1853. [CrossRef] [PubMed]

7. Sansone, R.; Rodriguez-Mateos, A.; Heuel, J.; Falk, D.; Schuler, D.; Wagstaff, R.; Kuhnle, G.G.; Spencer, J.P.; Schroeter, H.; Merx, M.W.; et al. Cocoa flavanol intake improves endothelial function and Framingham Risk Score in healthy men and women: A randomised, controlled, double-masked trial: The Flaviola Health Study. Br. J. Nutr. 2015, 114, 1246-1255. [CrossRef] [PubMed]

8. Dohadwala, M.M.; Holbrook, M.; Hamburg, N.M.; Shenouda, S.M.; Chung, W.B.; Titas, M.; Kluge, M.A.; Wang, N.; Palmisano, J.; Milbury, P.E.; et al. Effects of cranberry juice consumption on vascular function in patients with coronary artery disease. Am. J. Clin. Nutr. 2011, 93, 934-940. [CrossRef] [PubMed]

9. Rodriguez-Mateos, A.; Feliciano, R.P.; Boeres, A.; Weber, T.; Dos Santos, C.N.; Ventura, M.R.; Heiss, C. Cranberry (poly)phenol metabolites correlate with improvements in vascular function: A double-blind, randomized, controlled, dose-response, crossover study. Mol. Nutr. Food Res. 2016. [CrossRef] [PubMed]

10. Paixão, J.; Dinis, T.C.P.; Almeida, L.M. Malvidin-3-glucoside protects endothelial cells up-regulating endothelial NO synthase and inhibiting peroxynitrite-induced NF-kB activation. Chem. Biol. Interact. 2012, 199, 192-200. [CrossRef] [PubMed]

11. De Nigris, F.; Williams-Ignarro, S.; Lerman, L.O.; Crimi, E.; Botti, C.; Mansueto, G.; D'Armiento, F.P.; De Rosa, G.; Sica, V.; Ignarro, L.J.; et al. Beneficial effects of pomegranate juice on oxidation-sensitive genes and endothelial nitric oxide synthase activity at sites of perturbed shear stress. Proc. Natl. Acad. Sci. USA 2005, 102, 4896-4901. [CrossRef] [PubMed]

12. Edwards, M.; Czank, C.; Woodward, G.M.; Cassidy, A.; Kay, C.D. Phenolic Metabolites of Anthocyanins Modulate Mechanisms of Endothelial Function. J. Agric. Food Chem. 2015, 63, 2423-2431. [CrossRef] [PubMed]

13. Hidalgo, M.; Martin-Santamaria, S.; Recio, I.; Sanchez-Moreno, C.; De Pascual-Teresa, B.; Rimbach, G.; de Pascual-Teresa, S. Potential anti-inflammatory, anti-adhesive, anti/estrogenic, and angiotensin-converting enzyme inhibitory activities of anthocyanins and their gut metabolites. Genes Nutr. 2012, 7, 295-306. [CrossRef] [PubMed]

14. Mena, P.; Calani, L.; Bruni, R.; del Rio, D. Chapter 6-Bioactivation of High-Molecular-Weight Polyphenols by the Gut Microbiome. In Diet-Microbe Interactions in the Gut; Rio, K.T.D.D., Ed.; Academic Press: San Diego, CA, USA, 2015; pp. 73-101.

15. Larrosa, M.; García-Conesa, M.T.; Espín, J.C.; Tomás-Barberán, F.A. Ellagitannins, ellagic acid and vascular health. Mol. Asp. Med. 2010, 31, 513-539. [CrossRef] [PubMed] 
16. García-Muñoz, C.; Hernández, L.; Pérez, A.; Vaillant, F. Diversity of urinary excretion patterns of main ellagitannins' colonic metabolites after ingestion of tropical highland blackberry (Rubus adenotrichus) juice. Food Res. Int. 2014, 55, 161-169. [CrossRef]

17. De Nigris, F.; Balestrieri, M.L.; Williams-Ignarro, S.; D’Armiento, F.P.; Fiorito, C.; Ignarro, L.J.; Napoli, C. The influence of pomegranate fruit extract in comparison to regular pomegranate juice and seed oil on nitric oxide and arterial function in obese Zucker rats. Nitric Oxide Biol. Chem. 2007, 17, 50-54. [CrossRef] [PubMed]

18. Giménez-Bastida, J.A.; González-Sarrías, A.; Larrosa, M.; Tomás-Barberán, F.; Espín, J.C.; García-Conesa, M.T. Ellagitannin metabolites, urolithin A glucuronide and its aglycone urolithin A, ameliorate TNF- $\alpha$-induced inflammation and associated molecular markers in human aortic endothelial cells. Mol. Nutr. Food Res. 2012, 56, 784-796. [CrossRef] [PubMed]

19. Giménez-Bastida, J.A.; Larrosa, M.; González-Sarrías, A.; Tomás-Barberán, F.; Espín, J.C.; García-Conesa, M.T. Intestinal ellagitannin metabolites ameliorate cytokine-induced inflammation and associated molecular markers in human colon fibroblasts. J. Agric. Food Chem. 2012, 60, 8866-8876. [CrossRef] [PubMed]

20. González-Sarrías, A.; Azorín-Ortuño, M.; Yáñez-Gascón, M.J.; Tomás-Barberán, F.A.; García-Conesa, M.T.; Espín, J.C. Dissimilar in vitro and in vivo effects of ellagic acid and its microbiota-derived metabolites, urolithins, on the cytochrome P450 1A1. J. Agric. Food Chem. 2009, 57, 5623-5632. [CrossRef] [PubMed]

21. Gonzalez-Sarrias, A.; Gimenez-Bastida, J.A.; Nunez-Sanchez, M.A.; Larrosa, M.; Garcia-Conesa, M.T.; Tomas-Barberan, F.A.; Espin, J.C. Phase-II metabolism limits the antiproliferative activity of urolithins in human colon cancer cells. Eur. J. Nutr. 2014, 53, 853-864. [CrossRef] [PubMed]

22. González-Sarrías, A.; Núñez-Sánchez, M.; García-Villalba, R.; Tomás-Barberán, F.; Espín, J. Antiproliferative activity of the ellagic acid-derived gut microbiota isourolithin A and comparison with its urolithin A isomer: The role of cell metabolism. Eur. J. Nutr. 2015. [CrossRef] [PubMed]

23. González-Trujano, M.E.; Pellicer, F.; Mena, P.; Moreno, D.A.; García-Viguera, C. Antinociceptive and anti-inflammatory activities of a pomegranate (Punica granatum L.) extract rich in ellagitannins. Int. J. Food Sci. Nutr. 2015, 66, 395-399. [CrossRef] [PubMed]

24. Larrosa, M.; González-Sarrías, A.; Yáñez-Gascón, M.J.; Selma, M.V.; Azorín-Ortuño, M.; Toti, S.; Tomás-Barberán, F.; Dolara, P.; Espín, J.C. Anti-inflammatory properties of a pomegranate extract and its metabolite urolithin-A in a colitis rat model and the effect of colon inflammation on phenolic metabolism. J. Nutr. Biochem. 2010, 21, 717-725. [CrossRef] [PubMed]

25. Mele, L.; Mena, P.; Piemontese, A.; Marino, V.; López-Gutiérrez, N.; Bernini, F.; Brighenti, F.; Zanotti, I.; del Rio, D. Antiatherogenic effects of ellagic acid and urolithins in vitro. Arch. Biochem. Biophys. 2016, 599, 42-50. [CrossRef] [PubMed]

26. Sala, R.; Mena, P.; Savi, M.; Brighenti, F.; Crozier, A.; Miragoli, M.; Stilli, D.; del Rio, D. Urolithins at physiological concentrations affect the levels of pro-inflammatory cytokines and growth factor in cultured cardiac cells in hyperglucidic conditions. J. Funct. Foods 2015, 15, 97-105. [CrossRef]

27. Giorgio, C.; Mena, P.; del Rio, D.; Brighenti, F.; Barocelli, E.; Hassan-Mohamed, I.; Callegari, D.; Lodola, A.; Tognolini, M. The ellagitannin colonic metabolite urolithin D selectively inhibits EphA2 phosphorylation in prostate cancer cells. Mol. Nutr. Food Res. 2015, 59, 2155-2167. [CrossRef] [PubMed]

28. Han, Q.A.; Yan, C.; Wang, L.; Li, G.; Xu, Y.; Xia, X. Urolithin A attenuates ox-LDL induced endothelial dysfunction partly by modulating microRNA-27 and ERK/PPAR- $\gamma$ pathway. Mol. Nutr. Food Res. 2016. [CrossRef] [PubMed]

29. Michel, T.; Li, G.K.; Busconi, L. Phosphorylation and subcellular translocation of endothelial nitric oxide synthase. Proc. Natl. Acad. Sci. USA 1993, 90, 6252-6256. [CrossRef] [PubMed]

30. Montagnani, M.; Quon, M.J. Insulin action in vascular endothelium: Potential mechanisms linking insulin resistance with hypertension. Diabetes Obes. Metab. 2000, 2, 285-292. [CrossRef] [PubMed]

31. Kim, J.A.; Formoso, G.; Li, Y.; Potenza, M.A.; Marasciulo, F.L.; Montagnani, M.; Quon, M.J. Epigallocatechin gallate, a green tea polyphenol, mediates $\mathrm{NO}$-dependent vasodilation using signaling pathways in vascular endothelium requiring reactive oxygen species and Fyn. J. Biol. Chem. 2007, 282, 13736-13745. [CrossRef] [PubMed]

32. Xu, J.W.; Ikeda, K.; Yamori, Y. Upregulation of endothelial nitric oxide synthase by cyanidin-3-glucoside, a typical anthocyanin pigment. Hypertension 2004, 44, 217-222. [CrossRef] [PubMed] 
33. Tsikas, D. Methods of quantitative analysis of the nitric oxide metabolites nitrite and nitrate in human biological fluids. Free Radic. Res. 2005, 39, 797-815. [CrossRef] [PubMed]

34. Rodriguez-Mateos, A.; Toro-Funes, N.; Cifuentes-Gomez, T.; Cortese-Krott, M.; Heiss, C.; Spencer, J.P. Uptake and metabolism of (-)-epicatechin in endothelial cells. Arch. Biochem. Biophys. 2014, 559, 17-23. [CrossRef] [PubMed]

35. Mohan, M.; Waghulde, H.; Kasture, S. Effect of pomegranate juice on angiotensin II-induced hypertension in diabetic wistar rats. Phytother. Res. 2010, 24 (Suppl. S2), S196-S203. [PubMed]

36. Di Gesso, J.L.; Kerr, J.S.; Zhang, Q.; Raheem, S.; Yalamanchili, S.K.; O’Hagan, D.; Kay, C.D.; O’Connell, M.A. Flavonoid metabolites reduce tumor necrosis factor- $\alpha$ secretion to a greater extent than their precursor compounds in human THP-1 monocytes. Mol. Nutr. Food Res. 2015, 59, 1143-1154. [CrossRef] [PubMed]

37. Biesinger, S.; Michaels, H.A.; Quadros, A.S.; Qian, Y.; Rabovsky, A.B.; Badger, R.S.; Jalili, T. A combination of isolated phytochemicals and botanical extracts lowers diastolic blood pressure in a randomized controlled trial of hypertensive subjects. Eur. J. Clin. Nutr. 2016, 70, 10-16. [CrossRef] [PubMed]

38. Cerdá, B.; Espín, J.C.; Parra, S.; Martínez, P.; Tomás-Barberán, F.A. The potent in vitro antioxidant ellagitannins from pomegranate juice are metabolised into bioavailable but poor antioxidant hydroxy-6H-dibenzopyran-6-one derivatives by the colonic microflora of healthy humans. Eur. J. Nutr. 2004, 43, 205-220. [CrossRef] [PubMed]

39. Tomas-Barberan, F.A.; Gonzalez-Sarrias, A.; Garcia-Villalba, R.; Nunez-Sanchez, M.A.; Selma, M.V.; Garcia-Conesa, M.T.; Espin, J.C. Urolithins, the rescue of 'old' metabolites to understand a 'new' concept: Metabotypes as a nexus between phenolic metabolism, microbiota dysbiosis and host health status. Mol. Nutr. Food Res. 2016. [CrossRef] [PubMed]

40. Piwowarski, J.P.; Kiss, A.K.; Granica, S.; Moeslinger, T. Urolithins, gut microbiota-derived metabolites of ellagitannins, inhibit LPS-induced inflammation in RAW 264.7 murine macrophages. Mol. Nutr. Food Res. 2015, 59, 2168-2177. [CrossRef] [PubMed]

41. Rizza, S.; Muniyappa, R.; Iantorno, M.; Kim, J.A.; Chen, H.; Pullikotil, P.; Senese, N.; Tesauro, M.; Lauro, D.; Cardillo, C.; et al. Citrus polyphenol hesperidin stimulates production of nitric oxide in endothelial cells while improving endothelial function and reducing inflammatory markers in patients with metabolic syndrome. J. Clin. Endocrinol. Metab. 2011, 96, E782-E792. [CrossRef] [PubMed]

42. Ndiaye, M.; Chataigneau, M.; Lobysheva, I.; Chataigneau, T.; Schini-Kerth, V.B. Red wine polyphenol-induced, endothelium-dependent NO-mediated relaxation is due to the redox-sensitive PI3-kinase/Akt-dependent phosphorylation of endothelial NO-synthase in the isolated porcine coronary artery. FASEB J. 2005, 19, 455-457. [CrossRef] [PubMed]

43. Nuñez-Sanchez, M.A.; Garcia-Villalba, R.; Monedero-Saiz, T.; Garcia-Talavera, N.V.; Gomez-Sanchez, M.B.; Sanchez-Alvarez, C.; Garcia-Albert, A.M.; Rodriguez-Gil, F.J.; Ruiz-Marin, M.; Pastor-Quirante, F.A.; et al. Targeted metabolic profiling of pomegranate polyphenols and urolithins in plasma, urine and colon tissues from colorectal cancer patients. Mol. Nutr. Food Res. 2014, 58, 1199-1211. [CrossRef] [PubMed]

44. Dellafiora, L.; Mena, P.; Cozzini, P.; Brighenti, F.; Del Rio, D. Modelling the possible bioactivity of ellagitannin-derived metabolites. in silico tools to evaluate their potential xenoestrogenic behavior. Food Funct. 2013, 4, 1442-1451. [CrossRef] [PubMed]

45. Piwowarski, J.P.; Granica, S.; Kiss, A.K. Influence of gut microbiota-derived ellagitannins metabolites urolithins on pro-inflammatory activities of human neutrophils. Planta Med. 2014, 80, 887-895. [PubMed]

46. Kawai, Y.; Nishikawa, T.; Shiba, Y.; Saito, S.; Murota, K.; Shibata, N.; Kobayashi, M.; Kanayama, M.; Uchida, K.; Terao, J. Macrophage as a target of quercetin glucuronides in human atherosclerotic arteries: Implication in the anti-atherosclerotic mechanism of dietary flavonoids. J. Biol. Chem. 2008, 283, 9424-9434. [CrossRef] [PubMed]

47. Ishisaka, A.; Kawabata, K.; Miki, S.; Shiba, Y.; Minekawa, S.; Nishikawa, T.; Mukai, R.; Terao, J.; Kawai, Y. Mitochondrial Dysfunction Leads to Deconjugation of Quercetin Glucuronides in Inflammatory Macrophages. PLoS ONE 2013, 8, e80843. [CrossRef] [PubMed]

48. Tribolo, S.; Lodi, F.; Winterbone, M.S.; Saha, S.; Needs, P.W.; Hughes, D.A.; Kroon, P.A. Human metabolic transformation of quercetin blocks its capacity to decrease endothelial nitric oxide synthase (eNOS) expression and endothelin-1 secretion by human endothelial cells. J. Agric. Food Chem. 2013, 61, 8589-8596. [CrossRef] [PubMed] 
49. Dall'Asta, M.; Derlindati, E.; Curella, V.; Mena, P.; Calani, L.; Ray, S.; Zavaroni, I.; Brighenti, F.; del Rio, D. Effects of naringenin and its phase II metabolites on in vitro human macrophage gene expression. Int. J. Food Sci. Nutr. 2013, 64, 843-849. [CrossRef] [PubMed]

50. Tognolini, M.; Giorgio, C.; Hassan Mohamed, I.; Barocelli, E.; Calani, L.; Reynaud, E.; Dangles, O.; Borges, G.; Crozier, A.; Brighenti, F.; et al. Perturbation of the EphA2-ephrinA1 system in human prostate cancer cells by colonic (Poly)phenol catabolites. J. Agric. Food Chem. 2012, 60, 8877-8884. [CrossRef] [PubMed]

Sample Availability: Not available.

(C) 2016 by the authors; licensee MDPI, Basel, Switzerland. This article is an open access article distributed under the terms and conditions of the Creative Commons Attribution (CC-BY) license (http://creativecommons.org/licenses/by/4.0/). 